Laryng. Rhinol. Otol. 62 (1983) 586-589

(c) 1983 Georg Thieme Verlag Stuttgart · New York

\section{Tonsillenkarzinome und Epstein-Barr-Virus*}

\author{
E. Wilmes ${ }^{1}$, H. Wolf ${ }^{2}$, M. Haus $^{2}$ \\ Klinik und Poliklinik für HNO-Kranke ${ }^{1}$ \\ (Direktor: Professor Dr. H. H. Naumann) \\ Max-von-Pettenkofer-Institut ${ }^{2}$ (Direktor: Professor Dr. F. Deinhardt) der Lud- \\ wigs-Maximilians-Universität München
}

\section{Einleitung}

Das Epstein-Barr-Virus (EBV) ist der Erreger der infektiösen Mononukleose. Für den Kliniker und Virologen ist dieses zur Herpes-Gruppe zählende Virus besonders interessant, da es mit zwei humanen Neoplasien,

1. dem afrikanischen Burkitt-Lymphom (7) und

2. dem Nasopharynxkarzinom (21)

in ursächlichen Zusammenhang gebracht wird.

Primär stützte sich die Annahme einer viralen Genese dieser Tumoren auf sero-epidemiologische Studien $(11,12,13$, 23) und später auch auf Nukleinsäure-Hybridisierungen zum Nachweis viraler DNS in den Tumoren $(1,22,36)$.

Die Frage, ob diese virale DNS aus den Tumorzellen selbst oder aus den Lymphozyten, die häufig in diesen Tumoren zu finden sind, stammt, wurde mit Hilfe von in-situ-Hybridisierungen gelöst. Demnach ist die virale DNS in den epitheloiden Zellen der Karzinome lokalisiert (5, 29, 30). Bezüglich der onkogenen Eigenschaften eines Virus erfüllt das Epstein-Barr-Virus die Koch-Henleschen Postulate:

1. Tumorpatienten zeigen eine bis zu 10fache Titererhöhung von Antikörpern gegen EBV-spezifische Antigene (dies ist sowohl beim Burkitt-Lymphom als auch beim Nasopharynx-Karzinom der Fall) (15).

2. IgA-Antikörper gegen Virus-Kapsid-Antigen (VCA) und early antigen (EA) sprechen für einen $\mathrm{EBV}$-assoziierten Tumor $(14,27,28)$.

3. Die virale DNS kann in den Tumorzellen selbst nachgewiesen werden $(29,30)$.

4. EBV kann menschliche Zellen in vitro immortalisieren und in Primaten maligne Lymphome erzeugen (25).

5. Aus diesen Tumoren kann wiederum EBV-DNS isoliert werden (31).

6. Die Tumorzellen enthalten das virusspezifische Kernantigen (EBNA) $(18,20)$.

Bereits früher vertraten wir die Ansicht (27), daß das Epstein-Barr-Virus auch mit anderen Tumoren des lymphoepithelialen Rachenringes assoziiert sein könnte. Sie stützte sich auf serologische Untersuchungen, bei denen wir EBVspezifische Antikörper-Spektren bei verschiedenen Tumoren des Waldeyerschen Rachenringes fanden, die denen von Patienten mit Nasopharynxkarzinomen ähnelten. Es handelte sich um lymphoepitheliale und undifferenzierte Karzinome der Tonsillen, des Gaumens und des Zungengrundes.

*Herrn Prof. Dr. med. H. H. Naumann zum 65. Geburtstag gewidmet

\section{Zusammenfassung}

Das Epstein-Barr-Virus ist eng assoziiert mit Karzinomen des Nasopharynx (NPC). Die Verknüpfung des NPC mit dem EB-Virus beruht im wesentlichen auf dem gehäuften Nachweis von Antikörpern bei Patienten mit NPC im Vergleich mit Normalpersonen oder Patienten mit anderen malignen Tumoren.

Andererseits konnte das Virusgenom in Zellen von Nasopharynxkarzinomen mit Hilfe der Hybridisierungstechnik nachgewiesen werden.

Serologische Untersuchungen an unserer Klinik geben erste Hinweise darauf, daß auch ein Teil anderer Tumoren des Waldeyerschen Rachenrings ebenfalls mit EBV assoziiert sein kann.

Nukleinsäurehybridisierungen an 2 gering differenzierten Tonsillenkarzinomen konnten EBV-DNS auch in den Tumorzellen selbst nachweisen. Es ist offensichtlich, daß diese Tumoren in ähnlicher Weise wie das NPC mit EBV verknüpft sind.

Die ätiologische Bedeutung von EBV bei der Entstehung von Karzinomen im Waldeyerschen Rachenring wird diskutiert.

\section{Carcinoma of the Tonsils and Epstein-Barr Virus}

Epstein-Barr Virus is an important aetiological factor in the development of nasopharyngeal carcinoma (NPC).

Serology suggested that besides NPC some other tumours located within the lymphoepithelial ring of the throat (Waldeyer's ring) might be associated with EBV. Nucleic-acid hybridizations were carried out to detect the presence of EBV-DNA in tumour biopsies derived from tonsillar carcinomas.

We were able to demonstrate the presence of EBVDNA in the tumour cells of two poorly differentiated carcinomas. We think that only a small fraction of tonsillar carcinomas is positive for EBV. The aetiologic relationship of EBV with tumours of Waldeyer's ring is discussed.

Die EBV-Serologie war zwar ein Hinweis auf einen EBVassoziierten Tumor, sie reichte jedoch nicht für einen Beweis aus.

Als wichtigstes Indiz fehlte der Nachweis viraler DNS in den Tumorzellen selbst. Zur Beantwortung dieser Frage führten wir Untersuchungen an den Tumoren selbst durch.

\section{Material und Methodik}

Von Patienten mit Karzinomen der Tonsille wurden Probeexzisionen entnommen und bis zur späteren Verarbeitung bei $-40^{\circ} \mathrm{C}$ aufbewahrt.

\section{Prinzip der Nukleinsäure-Hybridisierungen}

Da nicht angenommen werden kann, daß das EBV als vollständiges Virus, sondern nur in Form seiner Nukleinsäure (DNS) vorliegt, reichen herkömmliche Nachweismethoden wie Histologie, Elektronenmikroskopie und Immunfluoreszenz nicht aus. Zum Nachweis der 


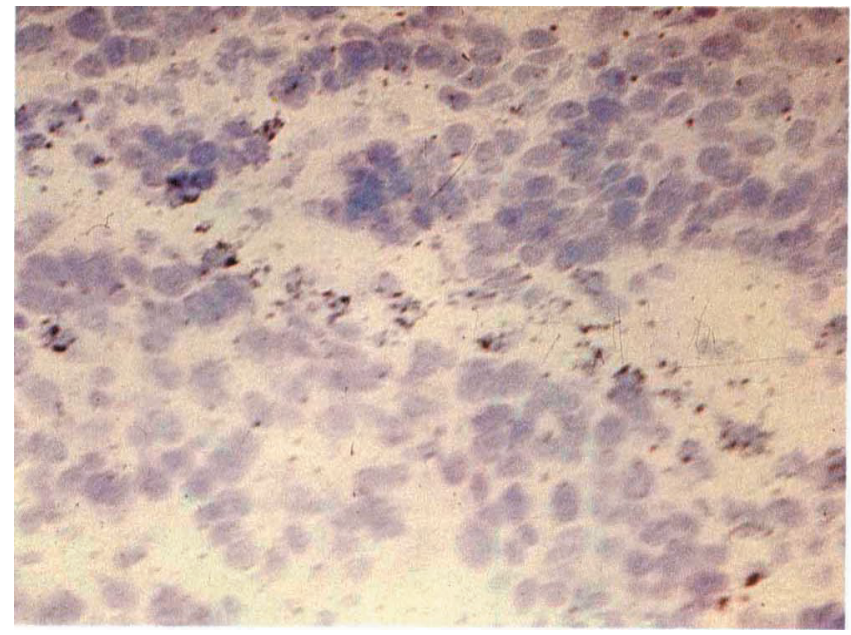

Abb. 1 Pat. B. J., gering diff. Tonsillenkarzinom (in-situ-Hybridisierung).

viralen DNS von EBV wurden daher Nukleinsäure-Hybridisierungen angewandt.

Hierzu wird zunächst eine radioaktiv-markierte virale DNS hergestellt. Diese wird entweder aus Virionen einer Virus produzierenden Zellinie (P3-HR1), die einem Burkitt-Lymphom entstammt, gewonnen, oder mit noch höherer Reinheit nach gentechnologischer Verknüpfung mit bakterieller DNS aus EBV-DNS-haltigen Bakterienplasmiden. Diese virale DNS wird dann mit verschiedenen Methoden radioaktiv markiert. Die DNS der Gewebe wird direkt in Gefrierschnitten durch Hitze oder Alkalibehandlung in Einzelstränge zerlegt und mit der markierten viralen DNS inkubiert. Die zugegebene virale DNS wird nur an die DNS der Präparate gebunden, wenn diese entsprechende Sequenzen viraler DNS enthalten (in-situ-Hybridisierung). Der Nachweis der Bindung erfolgt autoradiographisch durch Beschichtung der Schnitte mit Fotoemulsion.

\section{Durchführung der in-situ-Hybridisierung}

Zur in-situ-Hybridisierung (modifiziert nach Wolf und Mitarb. 29, 31) wurden $8 \mu \mathrm{m}$ dicke Gefrierschnitte $2 \mathrm{~min}$ in drei Teilen Methanol, einem Teil Eisessig fixiert, $30 \mathrm{~min}$ bei $70^{\circ} \mathrm{C}$ in $2 \times 5 S C(1 \times S S C=$ $0,15 \mathrm{M} \mathrm{NaCl}, 0,015 \mathrm{M} \mathrm{Na}$-Zitrat) inkubiert und $15 \mathrm{~min}$ bei $37^{\circ} \mathrm{C} \mathrm{mit}$ $\mu \mathrm{g} / \mathrm{ml}$ Proteinase-K behandelt. Direkt vor der Hybridisierung wurden die Präparate $3 \mathrm{sec}$ bei $98^{\circ} \mathrm{C}$ in $0,1 \times \mathrm{SSC}$ denaturiert, in dem gleichen Puffer bei $0^{\circ} \mathrm{C}$ abgeschreckt und luftgetrocknet.

$10 \mu \mathrm{l}$ Hybridisierungslösung (mit $100000 \mathrm{Ipm}$ radioaktiver EBVDNS, spezifisch aktiviert ca. $5 \times 10^{6} \mathrm{Ipm} / \mu \mathrm{g}$; in $50 \%$ Formamid $0,6 \mathrm{~m}$ $\mathrm{NaCl}, 10 \mathrm{mM}$ Tris-Hcl pH 7,5, $1 \mathrm{mM}$ EDTA, $100 \mu \mathrm{g} / \mathrm{ml}$ Kalbsthymus DNS, $1 \mathrm{mg} / \mathrm{ml}$ Rinderserumalbumin, $1 \mathrm{ml} / \mathrm{ml} \mathrm{t}-\mathrm{RNA}, 100 \mu \mathrm{g} / \mathrm{ml}$ Poly A, $0,02 \% \mathrm{wt} / \mathrm{vol}$ Polyvinylpyrrolidon, $0,02 \% \mathrm{wt} / \mathrm{vol}$ Ficoll) wurden unter einem silikonisierten Deckglas über den Schnitten mit Klebstoff eingeschlossen. Die Hybridisierung wurde über $4 \mathrm{Std}$. bei $4^{\circ} \mathrm{C}$ durchgeführt. Anschließend wurden die Deckgläser entfernt und die Schnitte mit fünffachem Wechsel von Formamidpuffer (wie Hybridisierungslösung, aber ohne Polyanionen) für $15 \mathrm{Std}$. gewaschen, mit $2 \times \mathrm{SSC}$ gespült und luftgetrocknet.

Diese Präparate wurden dann in Kodak-NTB-Emulsion (1:1 mit $6 \mathrm{mM}$ Ammoniumazetat verdünnt, $40^{\circ} \mathrm{C}$ ) getaucht, getrocknet und ca. 3 Wochen bei $4^{\circ} \mathrm{C}$ exponiert. Nach dem Entwickeln mit Kodak-D-19Röntgenfilmentwickler wurden die Präparate mit Giemsa gefärbt.

\section{Ergebnisse}

\section{Serologie}

Bei den hier vorgestellten Tonsillenkarzinomen handelte es sich histologisch um zwei gering differenzierte Plattenepithelkarzinome.

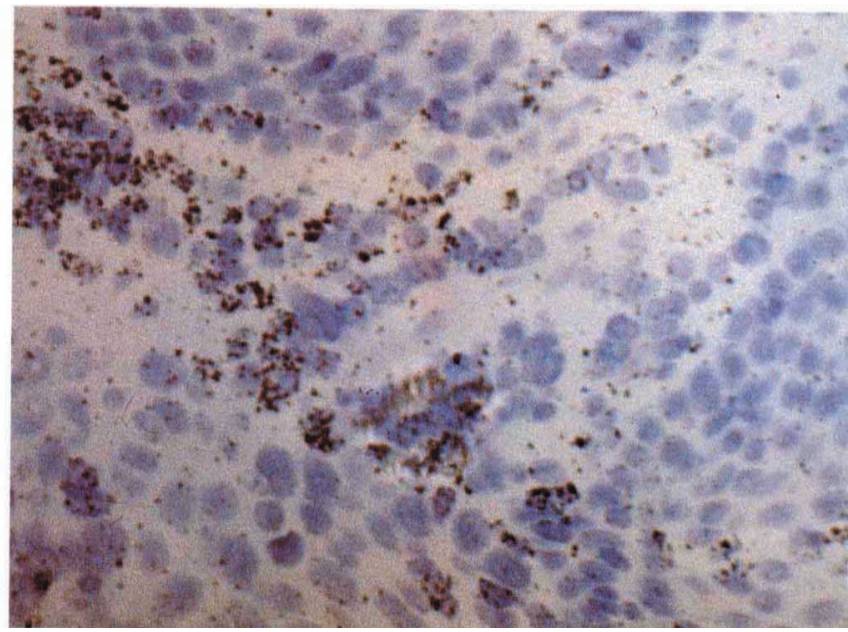

Abb. 2 Pat. St. A., gering diff. Tonsillenkarzinom (in-situ-Hybridisierung)

Die Antikörpertiter gegen EBV-Antigene sind aus der Tab. 1 zu entnehmen.

Tab. 1 Antikörpertiter gegen EBV-spezifische Antigene.

VCA: virus capsid antigen

$E A$ : early antigen

EBNA: EB-nuclear antigen

Pat. St. A. (21. 1. 1917) Pat. B. J. (3. 8. 1921)

\begin{tabular}{lll}
\hline EBV-VCA (lgA) & $1: 64$ & $1: 16$ \\
EBV-VCA (IgG) & $1: 64$ & $1: 32$ \\
EBV-VCA (IgA) & neg. & $1: 16$ \\
EBV-EA (lgG) & $1: 16$ & neg. \\
EBNA & $1: 16$ & $1: 16$
\end{tabular}

Wie beim Nasopharynxkarzinom zeigen oben genannte Tonsillenkarzinome die typischen IgA-Antikörper gegen das Virus-Kapsid-Antigen von Epstein-Barr-Virus. Drei weitere lymphoepitheliale Karzinome der Tonsillen wiesen keine IgA-(VCA)Antikörper im Serum auf. Gleiche serologische Befunde fanden sich bei 12 weiteren Tonsillenkarzinomen, die histologisch ein gering differenziertes Plattenepithelkarzinom aufwiesen. Die IgG-Antikörper liegen im Normbereich gesunder seropositiver Personen.

\section{In-situ-Hybridisierung}

Die autoradiographischen Bilder an Gefrierschnitten zeigen die Ergebnisse unserer in-situ-Hybridisierungen bei den oben genannten Tonsillenkarzinomen (Abb. 1 und 2).

Die geschwärzten Granula gelten als Nachweis viraler DNS, da sie durch radioaktiven Zerfall von spezifisch gebundener viraler Test-DNS erzeugt wurden. Hier haben sich Doppelstränge aus radioaktiv markierter viraler DNS und der DNS der Gewebe, die entsprechende Sequenzen enthält, gebildet.

Zellgrenzen sind nicht zu erkennen, da diese bei der Präparation weitgehend verloren gehen; allerdings sind die atypischen blasigen Zellkerne der gering differenzierten Karzinomzellen noch angedeutet zu erkennen. Dabei ist festzuhalten, daß es sich bei den markierten Zellen nicht um Lymphozyten handelt. 
Bei Kontrolluntersuchungen an normalen Gaumenmandeln (26), die ja vor allem aus Lymphozyten bestehen, konnten wir mit unserer Methode keine virale DNS nachweisen.

\section{Diskussion}

Die Ergebnisse unserer Untersuchungen zeigen, daß ein geringer Teil der Tonsillenkarzinome in ähnlicher Weise mit EBV assoziiert ist wie Nasopharynxkarzinome. Gemeinsam sind ihnen die gleichen EBV-spezifischen serologischen Parameter, beide enthalten Epstein-Barr-VirusDNS in den Tumorzellen.

$\mathrm{Zu}$ ähnlichen Ergebnissen kamen Brichacek und Mitarb. (4) mit einer anderen Methode. Allerdings konnte diese Arbeitsgruppe die EBV-Genome nur in der gesamten DNS der Gewebe nachweisen, nicht jedoch einem bestimmten Zelltyp zuordnen. So ist der wichtigste Einwand, daß die nachgewiesene virale DNS aus den Lymphozyten stammen könne, erst durch diese Arbeit widerlegt worden.

Diesen Ergebnissen entsprechen auch unsere Kontrolluntersuchungen an lymphatischen Geweben wie den Tonsillen, bei denen wir keine virale DNS nachweisen konnten (26).

Da die meisten normalen Tonsillen von uns nur mit der Technik der Reassoziationskinetik untersucht worden sind, ist allerdings nicht auszuschließen, daß mit der in-situ-Hybridisierung vereinzelt Lymphozyten, die EBV produzieren, nachweisbar wären, und damit ein Problem zur Abgrenzung gegenüber dem Gehalt an EBV-DNS in Tumorzellen stellen, Entsprechende Untersuchungen sind in Vorbereitung.

Zwischen den Tonsillenkarzinomen und den Nasopharynxkarzinomen besteht jedoch ein wichtiger Unterschied, der zur Zeit noch nicht erklärt werden kann.

Während alle bisher untersuchten undifferenzierten bzw. lymphoepithelialen Karzinome des Nasopharynx virale DNS enthalten und auch die typischen EBV-Antikörper im Serum nachweisbar sind, ist dies bei den Tonsillenkarzinomen nicht der Fall. So zeigten z. B. drei weitere lymphoepitheliale Karzinome der Tonsillen keine IgA-(VCA)Antikörperbildung bzw. erhöhte IgG-(VCA)Titer. Weitere 12 Tonsillenkarzinome, bei denen es sich histologisch um differenzierte Plattenepithelkarzinome handelte, zeigten ebenfalls wie die differenzierten Plattenepithelkarzinome des Nasopharynx eine negative Serologie. Zu ähnlichen Ergebnissen kamen kürzlich auch Sesterbenn und Mitarb. (24) bei der Untersuchung der lymphoepithelialen Karzinome des Oropharynx. Es scheint nur ein geringer Teil der wenig differenzierten Karzinome EBV-assoziiert zu sein.

Die Erklärung, warum gerade Nasopharynxkarzinome und auch Tonsillenkarzinome - beides Tumoren des Waldeyerschen Rachenringes - EBV-korreliert sind, mag sowohl in der gemeinsamen Entwicklungsgeschichte als auch in der ähnlichen Histologie zu finden sein.

Nach Döbnert (6) entstehen diese Geschwülste in einem lymphoepithelialen Gewebe, das gekennzeichnet ist durch die innige Durchdringung von Lymphozyten und epithelialen Zellen. Dies ist eine wichtige Voraussetzung für die Entstehung eines EBV-korrelierten Tumors $(2,3)$.

Als ein weiterer Faktor wird die exponierte Lage des Nasopharynxkarzinoms im Nasen-Rachenraum angesehen. Das Gewebe im Nasopharynx oder auch Oropharynx ist aufgrund dieser Lokalisation über Jahre oder Jahrzehnte den unterschiedlichsten pflanzlichen und chemischen Faktoren ausgesetzt $(16,17,19)$. Aus experimentellen Untersuchungen weiß man, daß diese Substanzen in der Lage sind, latent vorhandene virale EBV-DNS zu aktivieren, d. h. einen lytischen Zyklus in Gang zu setzen.

Ein lytischer Zyklus der Virusvermehrung führt jedoch bei DNS-haltigen Viren stets zum Absterben der entsprechenden Zellen und schließt somit die Entstehung von immortalisierten Zellen als Voraussetzung eines Tumors zumindest auf diesem direkten Weg aus. Um die scheinbare Diskrepanz zwischen der Induktion lytischer Virusfunktion gegenüber der für die Tumorentstehung verantwortlichen Transformation zu erklären, wurde ein alternatives Modell zur Tumorentstehung in mehreren Publikationen schrittweise hergeleitet $(3,32-36)$ und soll hier zusammenhängend kurz dargestellt werden.

Pflanzliche und chemische Induktoren treffen im Waldeyerschen Rachenring auf mit EBV latent infizierte Lymphozyten, welche sich bei über $90 \%$ der Bevölkerung als Folge einer primären Infektion finden. Diese Induktoren vermögen in den Lymphozyten EBV-spezifische Gene anzuschalten, wobei virale Proteine gebildet werden. Diese neu gebildeten Proteine sind experimenteller Untersuchungen zufolge in der Lage, eine Zellfusion zwischen Lymphozyten und benachbarten Zellen herbeizuführen unter der Voraussetzung, daß die Zellen in engem Kontakt miteinander stehen $(2,3)$. Oben genannte Voraussetzungen, nämlich die exponierte Lage und der enge Kontakt zwischen Lymphozyten und Epithelzellen sind im Waldeyerschen Rachenring gegeben.

Auf dem Wege der Zellfusion könnte die virale DNS aus den Lymphozyten in epitheliale Zellen gelangen, die keine nachweisbaren Rezeptoren für EBV besitzen. In diesen fusionierten Zellen scheint der lytische Zyklus von EBV, der durch Induktoren mit der Bildung früher Antigene in Gang gesetzt worden ist, unterdrückt zu werden $(9,10)$.

Die auf indirektem Wege infizierte Zelle geht also nicht in einem lytischen Zyklus zugrunde, sondern proliferiert aufgrund der Information durch das virale Genom.

Die Mehrzahl dieser Zellen mit Proliferationspotential wird entweder durch immunologische Mechanismen eliminiert oder in ihrem Wachstum kontrolliert. Entkommt aber nach entsprechender Selektion ein Zellklon der Immunabwehr, so kann ein monoklonaler Tumor (8), ein Nasopharynxkarzinom entstehen.

Obwohl einige der geschilderten Schritte zur Karzinogenese eines EBV-korrelierten Tumors noch hypothetisch sind und auf in vitro durchgeführten Untersuchungen basieren, so können sie unseres Erachtens als Ausgangspunkt und Modell für weitere Untersuchungen dienen.

\section{Literatur}

(1) Anderson-Anvret, M., N. Forsby, G. Klein, G. Henle: Relationship between the EBV and undifferential nasopharyngeal carcinoma: Correlated nucleic acid hybridisation and histopathological examination. Int. J. Cancer 20 (1977) 486

(2) Bayliss, G. J., H. Wolf: EpsteinBarr virus-induced cell fusion. Nature, 287 (1980) 164-165

(3) Bayliss, G. J., H. Wolf: An EpsteinBarr virus early protein induced cell fusion. Proc, Natl, Acid, Sci. (USA) 78 (1981) 7162

(4) Brichacek, B, A. Suchankova, I. Hirsch, O, Sibl, D, Rezacova, H, Zavadova, $V$. Vonka: Presence of $E_{p}-$ stein-Barr Virus DNA in tonsillar tissues. Acta virol. 25 (1981) 361
(5) Desgranges, C., H. Wolf, G. de The, K. Shanmugaratnam, N. Cammoun, R. Ellouz, G, Klein, K. Lennert, N. Nunoz, H. zurHausen: Nasopharyngeal carcinoma, X. Presence of Epstein-Barr genomes in separated epithelial cells of tumours in patients from Singapore, Tunisia and Kenya. Int. J. Cancer 16 (1975) 7

(6) Döhnert, G.: Über lymphoepitheliale Geschwülste, Erkenntnisse anhand der Gewebekultur und vergleichender klinischer, morphologischer und virologischer Untersuch ungen. In: Sitzungsbericht der Heidelberger Akademie der Wissenschaften. 3. Abhandlung. Springer Verlag Berlin, Heidelberg, New York (1977).

(7) Epstein, M. A., B. G. Achong: The Relationship of the virus to Burkitt's 
lymphoma. In: M. A. Epstein und B. G. Achong (eds.): The Epstein-Barr Virus. Springer Verlag, Berlin-Heidelberg 1979

(8) Fialkow, P. J, G. M. Martin, G. Klein, P. Clifford, S. Singh: Evidence for a clonal origin of head and neck tumours. Int. J. Cancer 9 (1972) 133 (9) Glaser, R., J. Zimmermann, S. St. Jeor, F. Rapp: Demonstration of a cellular inhibitor of Epstein-Barr and cytomegalovirus synthesis. Virology 64 (1975) 89

(10) Graessmann, A., H. Wolf, G. W. Bornkamm: Expression of EpsteinBarr virus genes in different cell types after microinjection of viral DNA. Proc. Natl. Acad. Sci. USA, 77 (1980) 433

(11) Henle, G., W. Henle: Immunofluorescense in cells derived from Burkitt's lymphoma. J. Bact. 91 (1966) 1248

(12) Henle, W., G. Henle, H. C. Ho, P. Bartu, Y. Cachin, P. Clifford, A. De Schryver, G. de The, V. Diehl, G.

Klein: Antibodies to EBV in nasopharyngeal carcinoma, other head and neck neoplasms and control groups. $J$ Nat. Cancer Inst. 44 (1970 a) 225

(13) Henle, W., H. C. Ho, G. Henle, J. C. Kwan: Antibodies to Epstein-Barr virus related antigens in nasopharyngeal carcinoma. Comparison of active disease and long term survivors. J. Nat. Cancer Inst. 51 (1973) 361

(14) Henle, G., W. Henle: EpsteinBarr virus specific IgA serum antibodies as an outstanding feature of nasopharyngeal carcinoma. Int. J. Cancer 17 (1976) 1

(15) Henle, G., W. Henle: Seroepidemiology of the virus. In: Epstein, $M$. A., B. G. Achong (eds). The EpsteinBarr virus. Springer-Verlag, BerlinHeidelberg-New York (1979) 61

(16) Ho, J. H. C.: Genetic and environmental factors in nasopharyngeal carcinoma. In: Recent advances in hu- man tumour virology and immunology (Proceedings of the first international cancer symposium of the Princess Takamatsu cancer research fund $(\mathrm{Na}$ kahara, Nishioka, Hirayama, Ito, eds.) University of Tokyo Press, Tokyo (1971) 275

(17) Ho, J. H. C.: Nasopharyngeal carcinoma (NPC), in: Advances in cancer research (Klein, Weinhouse, Haddow, eds.) Academic Press, New York and London (1972) 57

(18) Huang, D. P., H. C. Ho, E. Henle, G. Henle: Demonstration of EBV-associated nuclear antigens in NPC cells from fresh biopsies. Int. J. Cancer 14 (1974) 580

(19) Ito, Y., M. Kishiskita, T. Moriga$\mathrm{ki}, \mathrm{S}$. Yanase, T. Hirayama: Induction and intervention of Epstein-Barr virus expression in human lymphoblastoid cell lines: A simulation model for study of cause and prevention of nasopharyngeal carcinoma and Burkitt's

lymphoma. Grundmann et al. (eds.) Cancer Campaign, Vol 5, Nasopharyngeal carcinoma, G. Fischer Verlag, Stuttgart-New York 1981

(20) Klein, G., B. Biovanella, T. Lindahl, P. Fialkow, S. Singh, J. Stehlin: Direct evidence for the presence of EBV-DNA and nuclear antigen in malignant epithelial cells from patients with anaplastic carcinoma of the nasopharynx. Proc. Natl. Acad. Sci. (USA) 71 (1974) 4737

(21) Klein, G.: The relationship of the virus to nasopharyngeal carcinoma. In: Epstein, Achong (eds.): The Epstein-Barr virus. Springer, Berlin-Heidelberg 1979

(22) Nonoyama, G., C. Huang, J. Pagano, G. Klein, S. Singh: DNA of Epstein-Barr virus detected in tissue of Burkitt's lymphoma and nasopharyngeal carcinoma. Proc. Natl. Acad. Sci. (USA) 70 (1973) 3265

(23) Old, L., E. Boyse, H. Oettgen, E. De Harven, G. Geering, B. William- son, P. Clifford: Precipitating antibody in human serum to an antigen present in cultured Burkitt's lymphoma cells. Proc. Natl. Acad. Sci. (Wash.) 56 (1966) 1699

(24) Sesterhenn, K., G. Krueger, G. Bertram, I. Sesterhenn: Zum lympho epithelialen Karzinom des Oropharynx. HNO 30 (1982) 243

(25) Shope, T., D. Dechairo, G. Miller: Malignant lymphoma in cotton top marmosets following inoculation of Epstein-Barr virus. Proc. Natl. Acad. Sci. (USA) 70 (1973) 2487 (26) Wilmes, E., H. Wolf: Der Nachweis von Epstein-Barr-Virus Genomen in der Orhspeicheldrüse. Laryng. Rhinol. 60 (1981) 7

(27) Wilmes, E., H. Wolf, F. Deinhardt, H. H. Naumann: EBV-Serology in NPC and related malignancies. Cancer Campaign, Vol. 5, Nasopharyngeal carcinoma, Grundmann et al. (eds.), Gustav Fischer Verlag, Stuttgart-New York 1.981

(28) Wilmes, E., H. Wolf, F. Deinhardt, H. H. Naumann: Die Bedeutung von Epstein-Barr-Virus-Antikörpern für Diagnose und Verlauf des Nasopharynxkarzinoms. Laryng. Rhinol. 58 (1979) 911

(29) Wolf, H., H. zurHausen, V. Bekker: EB viral genomes in epithelial nasopharyngeal carcinoma cells. Nature New Biology 138 (1973) 245

(30) Wolf, H. H. zurHausen, G. Klein, V. Becker, G. Henle, W. Henle: Attempts to detect virus-specific DNA sequences in human tumours, III. Epstein-Barr viral DNA in non-Jymphoid nasopharyngeal carcinoma cells. Med. Microbiol. Immunol., 161 (1975) 15

Priv.-Doz. Dr. med. E. Wilmes Klinik und Poliklinik für HNO-Kranke Marcbioninistr. 15 8000 München 70
(31) Wolf H. J. Werner, H. zurHausen: EBV-DNA in non-lymphoid cells of nasopharyngeal carcinomas and in a malienant lymphoma obtained after inoculation of $\mathrm{EBV}$ into cotton-top marmosets. Cold Spring Harbor Symposium on Quantitative Biology, Vol. 39 (1975) 791

(32) Wolf, H., G. J. Bayliss, E. Wilmes: Biological properties of EpsteinBarr virus. Cancer Campaign, Vol. 5 , Nasopharyngeal carcinoma, Grundmann et al. (eds.), Gustav Fischer Verlag, Stuttgart-New York, 1981

(33) Wolf, H., E. Wilmes, G. J. Bayliss: Epstein-Barr Virus: Its Site of Persistence and its Role in the Development of Carcinomas. Haematology and Blood Transfusion Vol. 26, Modern Trends in Human Leukemia IV, 191.195 , Neth, Gallo, Graf, Mannweiler, Winkler (eds.), Springer BerlinHeidelberg 1981

(34) Wolf, H.: On the biology of Epstein-Barr virus. In: Human deficiency and Cancer: Epstein-Barr Virus on lymphoproliferative malignancies. David Purtilo, Ed., Plenum Press, New York (1983)

(35) Wolf, H., G. J. Bayliss, R. Seibl: New results on the biology of Epstein Barr virus. Current topics in veterinary medicine and animal science. (Willmann, G., A. Rziha, R. Geskell, M. Nijhoff (eds.), im Druck

(36) ZurHausen, H., H. Schulte-Holthausen, G. Klein, W. Henle, G. Henle, L. Santesson, P. Clifford: EBV-DNA in biopsies of Burkitt tumours and anaplastic carcinomas of the nasopharynx. Nature 228 (1970) 1056 